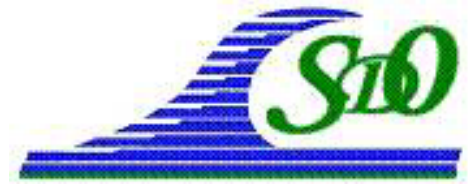

XI ìmes Journées Nationales Génie Côtier - Génie Civil

Les Sables d'Olonne, 22-25 juin 2010

DOI:10.5150/jngcgc.2010.037-G C Editions Paralia CFL

disponible en ligne - http://www.paralia.fr - available online

\title{
Simulation du clapage de sédiment avec un modèle à deux phases
}

\section{Sylvain GUILLOU ${ }^{1}$, Julien CHAUCHAT ${ }^{2}$, Damien PHAM VAN BANG ${ }^{3}$, Duc Hau NGUYEN ${ }^{1}$, Kim Dan NGUYEN ${ }^{3}$}

\section{Université de Caen, LUSAC (EA4253),}

Site Universitaire de Cherbourg, BP 78, 50130 Octeville, France.

sylvain.guillou@unicaen.fr

2. Université de Grenoble, LEGI, CNRS UMR 5519,

BP 53, F-38041 Grenoble Cedex 9, France.

julien.chauchat@hmg.inpg.fr

3. Université Paris-Est, LSHV, (Joint Research Unit EDF R\&D-CETMEF-ENPC),

6 quai Watier, BP 49, Bat. K, F-78401 Chatou, France.

damien.pham-van-bang@developpement-durable.gouv

\section{Résumé :}

La maintenance des chenaux de navigation et des zones portuaires implique la réalisation d'opérations de dragage pour garantir une profondeur d'eau suffisante. Les produits de dragages sont déposés en mer (opération de clapage) si les conditions d'immersion fixées par la convention d'Oslo sont satisfaites. Toutefois, le clapage de sédiments peut induire des nuisances sur l'environnement. Nous utilisons un modèle de transport sédimentaire à deux phases que nous avons développé (BARBRY et al., 2000 ; NGUYEN et al., 2009 ; CHAUCHAT et al., 2008) pour simuler le phénomène de clapage de sédiment fin. La comparaison des simulations aux résultats expérimentaux réalisés en canal par BOUTIN (2000), VILLARET et al. (1997) et VILLARET et al. (1998) montre le bon comportement du modèle pour décrire les différentes phases dynamiques de ce phénomène.

\section{Mots-clés :}

Clapage - Transport sédimentaire - Modélisation numérique - Modèle à deux phases

\section{Introduction}

La gestion des clapage (ou rejets en mer des produits de dragage) constitue une problématique environnementale et économique importante pour les gestionnaires des voies navigables et des infrastructures portuaires. Moins coûteuses qu'un stockage à terre, ces opérations peuvent induire des nuisances sur l'environnement notamment en provoquant un accroissement local de la turbidité et en ensevelissant les habitats de la faune aquatique.

Le phénomène de clapage comporte principalement trois phases (BOUTIN, 2000) : la phase de chute (soumise aux courants); l'impact sur le fond et la génération d'un 
courant de densité ; la propagation des courants de densité et la décantation des sédiments.

L'objet de ce travail est l'étude du phénomène de clapage par la simulation numérique. Des travaux antérieurs ont montré les limites à l'utilisation de codes basés sur l'hypothèse du scalaire passif dans cette configuration (GARAPON et al., 2002). Ceci est lié aux très fortes concentrations du rejet. Dans des travaux plus récents, FRESON (2004) a présenté un modèle bi-espèce pour le problème du clapage. Ce modèle est basé sur une dégénérescence du modèle bi-phasique à deux fluides. Il considère le mélange eau-sédiment dans son ensemble et ne tient pas compte de la rhéologie des sédiments cohésifs (FAROUT-FRESON et al., 2006). Si des résultats satisfaisants ont été obtenus pour la phase de chute, l'approche bi-espèce semble inappropriée pour décrire l'impact sur le fond, la génération et la propagation des courants de densité.

L'utilisation de l'approche à deux phases pour traiter la problématique hydrosédimentaire est apparue dans les années 90 (TEISSON et al., 1992 ; NGUYEN et $a l$., 2009, et références incluses). Dans cette approche le mélange eau/sédiment est défini comme constitué de deux phases : une phase continue, l'eau, et une phase dispersée, les sédiments. Des équations de conservation de la masse et de la quantité de mouvements sont écrites pour chaque phase. Le domaine d'étude s'étend du fond non érodable jusqu'à la surface libre. C'est-à-dire que les processus de transport des particules en suspension, de sédimentation, de tassement et de consolidation sont pris en compte de manière continue sans avoir à considérer l'érosion d'une couche ou le remplissage d'une autre. Le modèle à deux phases de transport sédimentaire que nous avons développé (BARBRY et al., 2000 ; NGUYEN et al., 2009, CHAUCHAT et al., 2008) est un modèle à deux fluides de ce type. Il est adapté aux milieux denses en différentiant les deux constituants et il inclut les effets de la turbulence. Dans ce travail, ce modèle est appliqué au cas du clapage. La configuration expérimentale utilisée par VILLARET et al. (1997) et VILLARET et al. (1998) a été retenu.

\section{Modèle à deux phases}

Dans l'approche à deux phases Eulérienne-Eulérienne (fluide - particule solide) ou à deux fluides, on considère que les mouvements de chaque phase sont régis respectivement par des équations de conservation de la masse et de la quantité de mouvements. Nous rappelons ici quelques éléments concernant le modèle utilisé, mais le lecteur pourra trouver une description complète du modèle dans les références suivantes : BARBRY et al. (2000), CHAUCHAT et GUILLOU (2008), NGUYEN et al. (2009).

\subsection{Les équations de bases}

En notant $k$, l'indice désignant la phase fluide ou la phase solide, les équations de conservation sont données : 


$$
\left\{\begin{array}{c}
\frac{\partial\left(\alpha_{k}\right)}{\partial t}+\vec{\nabla} \cdot\left(\alpha_{k} \overrightarrow{u_{k}}\right)=0 \\
\frac{\partial\left(\alpha_{k} \overrightarrow{u_{k}}\right)}{\partial t}+\vec{\nabla} \cdot\left(\alpha_{k} \rho_{k} \overrightarrow{u_{k}} \otimes \overrightarrow{u_{k}}\right)=\frac{1}{\rho_{k}} \vec{\nabla} \cdot\left(\alpha_{k}\left(-p_{k} \overline{\bar{I}}+\overline{\overline{\tau_{k}}}+\overline{\overline{\tau_{k}^{\mathrm{Re}}}}\right)\right)+\alpha_{k} \vec{g}+\frac{1}{\rho_{k}} \overrightarrow{M_{k}}
\end{array}\right.
$$

où $\alpha_{\mathrm{k}}$, $\vec{u}_{k}$, et $\rho_{k}$, représentent respectivement la fraction volumique, le vecteur vitesse et la masse volumique de la phase $k$ ( $k=f$ pour le fluide, $k=s$ pour le solide). $\vec{g}$ est le vecteur accélération de la pesanteur et $\vec{M}_{k}$ représente le transfert de quantité de mouvement entre les phases. $p_{k}$ est la pression de la phase $k, \overline{\overline{\tau_{k}}}$ et $\overline{\overline{\tau_{k}^{\mathrm{Re}}}}$ représentent respectivement le tenseur des contraintes visqueuses et le tenseur des contraintes de Reynolds pour la phase $k$. La somme des fractions volumiques est égale à 1. Le tenseur des contraintes visqueuses est modélisé en fonction des tenseurs des taux de déformation de chacune des phases ( $\overline{\overline{D_{f}}}$ et $\overline{\overline{D_{s}}}$ ) par la relation (2) (LUNDGREN, 1972) avec les coefficients de viscosité $\mu_{f f}, \mu_{f s}, \mu_{s f}$ and $\mu_{s s}$ donnés par (3). Le caractère non Newtonien est pris en compte par l'introduction du facteur d'amplification $\beta$, qui est lié à la distance interparticulaire $\xi$, et $d$ le diamètre des particules. Plus la distance interparticulaire est faible, plus le milieu est dense, et plus les frictions sont importantes. Nous utilisons la formulation (4) proposée par GRAHAM (1981), qui couvre les écoulements de suspensions diluées à denses. La distance interparticulaire est donnée par la relation (5) en fonction de la fraction volumique de la phase solide et sa valeur maximale $\alpha_{s, \max }$ (pour des particules non cohésives $\alpha_{s, \max }=0.625$ ).

$$
\begin{aligned}
& \alpha_{f} \overline{\overline{\tau_{f}}}=\mu_{f s} \overline{\overline{D_{s}}}+\mu_{f f} \overline{\overline{D_{f}}} \quad \alpha_{s} \overline{\overline{\tau_{s}}}=\mu_{s s} \overline{\overline{D_{s}}}+\mu_{s f} \overline{\overline{D_{f}}} \\
& \mu_{f f}=\alpha_{f} \mu_{f} \quad \mu_{f s}=\alpha_{s} \mu_{f} \quad \mu_{s s}=\alpha_{s} \beta \mu_{f s} \quad \mu_{s f}=\alpha_{s} \beta \mu_{f f} \\
& \beta=\frac{5}{2}+\frac{9}{4}\left(\frac{1}{1+\xi / d}\right)\left[\frac{1}{2 \xi / d}-\frac{1}{1+2 \xi / d}-\frac{1}{(1+2 \xi / d)^{2}}\right] \frac{1}{\alpha_{s}} \\
& \xi / d=\frac{1-\left(\alpha_{s} / \alpha_{s, \max }\right)^{1 / 3}}{\left(\alpha_{s} / \alpha_{s, \max }\right)^{1 / 3}} \\
& \vec{M}_{f}=p_{f i} \vec{\nabla} \alpha_{f}-\overline{\overline{\tau_{f i}}} \vec{\nabla} \alpha_{f}+\vec{M}_{f}^{\prime} \quad \vec{M}_{s}=p_{s i} \vec{\nabla} \alpha_{s}-\overline{\overline{\tau_{s i}}} \vec{\nabla} \alpha_{s}+\vec{M}_{s}^{\prime} \quad \vec{M}_{f}^{\prime}=-\vec{M}_{s}^{\prime}
\end{aligned}
$$

Le terme de transfert de quantité de mouvements entre les phases est évalué par la relation (6) en fonction de la pression et de la contrainte interfaciale $p_{k i}$ et $\overline{\overline{\tau_{k i}}}$ et de $\vec{M}^{\prime}{ }_{k}$ qui représente les différentes forces agissant sur la phase $k$ dont la force de traînée. $p_{f i}$ et $\tau_{k i}$ sont respectivement la pression et le tenseur de contrainte de cisaillement de la phase $k$ à l'interface. Les pressions interfaciales des deux phases ainsi que les tenseurs interfaciaux de contrainte tangentielle sont données par la relation (7). La pression de la phase solide est prise égale à la pression interfaciale de la même phase. 


$$
p_{f i}=p_{f}-\frac{\rho_{f}}{4}\left\|\overrightarrow{u_{f}}-\overrightarrow{u_{s}}\right\|^{2} \quad p_{s i}=p_{f i} \quad \overline{\overline{\tau_{s i}}}=\overline{\overline{\tau_{f i}}}=\beta \overline{\overline{\tau_{f}}}
$$

Le terme d'échange entre les phases $\vec{M}_{f}{ }^{\prime}=-\vec{M}_{s}$ ' est la somme des forces s'exerçant sur la particule solide. Ici seule la force volumique de traînée (relation (8)) est retenue (HSU et al., 2003). Elle s'exprime en fonction de $\vec{u}_{r}$, la vitesse relative entre le fluide et les particules, et de $\tau_{\text {fs }}$, le temps de relaxation de la particule. Ce dernier terme correspond au temps d'existence d'une différence de vitesse entre la particule solide et le fluide. Le coefficient $C_{D}$ est le coefficient de traînée moyen. Il dépend du nombre de Reynolds sédimentaire $R e_{s}$ et de la fonction de forme $\Psi$ de la particule solide. Nous utilisons la formule de HAIDER et LEVENSPIEL (1989).

$$
\vec{M}_{s}^{\prime} \approx \vec{F}_{D}=\frac{\alpha_{s} \rho_{s}}{\tau_{f s}} \vec{u}_{r} \quad \tau_{f s}=\frac{4 d \rho_{s}}{3 \rho_{f} C_{D}\left\|\vec{u}_{r}\right\|} \quad C_{D}=\frac{24}{\operatorname{Re}_{s}} f\left(\operatorname{Re}_{s}, \psi\right)
$$

La vitesse relative est définie comme $\vec{u}_{r}=\vec{u}_{s}-\vec{u}_{f}-\vec{u}_{d}$, où $\vec{u}_{d}=\left\langle\vec{u}_{f}^{\prime}\right\rangle_{s}$ est la vitesse de dérive qui représente la corrélation entre les fluctuations de vitesse de la phase fluide et la distribution spatiale instantanée des particules. Cette vitesse représente une manifestation des effets de la turbulence. Plusieurs modèles de turbulence ont été développés dans le code de calcul. Ceux-ci ne sont pas détaillé ici, mais le lecteur trouvera une présentation détaillé dans CHAUCHAT et GUILLOU (2008).

\subsection{Technique de résolution numérique}

Nous utilisons les techniques développées par GUILLOU et al. (2000) pour résolution du système d'équations (1). Le modèle est bidimensionnel vertical à surface libre. La technique de maillage en coordonnée $\sigma$ est utilisée pour suivre l'évolution de la surface libre à chaque instant. Les opérateurs de dérivées spatiales sont discrétisés par la technique des différences finies. Une technique de projection est utilisée pour le découplage du calcul de la vitesse de la phase fluide et de la pression. Les équations du modèle sont discrétisées de manière implicite sur la verticale et explicite sur l'horizontale. La méthode GMRES est utilisée pour l'inversion du système matriciel de pression. Un positionnement décalé et entrelacé des inconnues sur la grille de calcul est employé afin d'éviter les oscillations numériques qui risquent de se produire lors de l'utilisation de le technique de projection (GUILLOU \& NGUYEN, 1999).

\section{Configuration de l'étude}

De nombreux essais de clapage ont été réalisés dans le canal $\mathrm{n}^{\circ} 5$ du LNHE-EDF $(80 \mathrm{~m} \times 1.5 \mathrm{~m} \times 1.5 \mathrm{~m})$ par BOUTIN (2000), VILLARET et al. (1997) et VILLARET et al. (1998). Le but était d'étudier la phase de chute du rejet ainsi que la phase de courant de densité sur le fond, dans un fluide au repos ou en mouvement. Les tests ont été réalisés avec du sable, de la vase et des mélanges sablo-vaseux. Nous nous limitons ici au cas du sable. Nous présentons succinctement les conditions expérimentales 


\section{XI $I^{\text {èes }}$ Journées Nationales Génie Côtier - Génie Civil}

Les Sables d'Olonne, 22-25 juin 2010

utilisées par VILLARET et al. (1997) et VILLARET et al. (1998) sur lesquelles les simulations s'appuient.

\subsection{Configuration expérimentale de référence}

Le montage expérimental est constitué d'un canal équipé d'un dispositif d'injection de sédiment placé à $15 \mathrm{~cm}$ sous la surface libre et piloté par un PC. A $t=0$, un volume initial de mélange eau-sédiment à une concentration désirée est lâché dans l'eau. Une caméra synchronisée prend des clichés du panache turbide généré, pendant qu'un système enregistre la concentration en plusieurs points du canal. Le sable injecté est de densité $2650 \mathrm{~kg} / \mathrm{m}^{3}$, et de diamètre $\left(D_{p}\right) 90$ ou $160 \mu \mathrm{m}$. La concentration du rejet $(\mathrm{Cr})$ varie de 350 à $450 \mathrm{~g} / 1$ pour un volume initial $(V r)$ de sédiments de 45 ou 60 litres. Les paramètres clés sont le temps de chute du rejet (temps pour que le nuage de sédiment atteigne le fond), le diamètre du rejet pendant la phase de chute, la hauteur du front pendant la phase de transport sur le fond. L'incertitude sur le temps de chute est de 0.5 seconde, tandis que celle pour le diamètre du rejet est de $5 \mathrm{~cm}$.

Tableau 1. Caractéristiques des essais 100\% sable sans courant ambiant (VILLARET et al., 1997 ; VILLARET et al., 1998).

\begin{tabular}{llllll}
\hline Tests & $\boldsymbol{W}_{\boldsymbol{i n j} \boldsymbol{j}}(\mathbf{m} / \mathbf{s})$ & $\boldsymbol{D}_{\boldsymbol{p}}(\boldsymbol{\mu m})$ & $\boldsymbol{\rho}\left(\mathbf{k g} \cdot \mathbf{m}^{-\mathbf{3}}\right)$ & $\boldsymbol{C r}(\mathbf{g} / \mathbf{l})$ & $\boldsymbol{V r}(\boldsymbol{l})$ \\
\hline e6 & 0.6 & 90 & 2650 & 350 & 45 \\
el1 & 0.79 & 90 & 2650 & 450 & 60 \\
e12 & 0.89 & 160 & 2650 & 450 & 60 \\
\hline
\end{tabular}

\subsection{Paramétrage du modèle et méthode d'analyse}

Le domaine d'étude couvre une zone de 4 mètres horizontalement centrée sur le rejet et de 1 mètre en hauteur. Un maillage régulier de 401 nœuds sur l'horizontale et de 41 nœuds sur la verticale est utilisé. Le pas de temps est fixé à $\Delta t=0,001 \mathrm{~s}$ tandis que la concentration du rejet initial $(\mathrm{Cr})$ est donnée par le tableau 1. Un schéma UPWIND premier ordre est utilisé pour les termes de convection. Les frontières latérales sont considérées ouvertes pour permettre au rejet de se propager et de ne pas générer de réflexions. La surface libre est figée dans ces simulations. En conformité avec les conditions expérimentales, l'injection du mélange eau-sédiment est effectuée à $15 \mathrm{~cm}$ sous la surface libre et pour un diamètre de $10 \mathrm{~cm}$. La concentration y est imposée ainsi qu'un profil parabolique de vitesse de type Poiseuille dont la vitesse maximale $\left(W_{i n j}\right)$ est donnée par le modèle analytique de KRISHNAPPAN (1975). Les valeurs sont données dans le tableau 1 . On note que les résultats sont sensibles à la manière de réaliser l'injection. 


\section{Résultats}

La figure 1 présente les résultats de simulation à différents instants pour l'essai e6. Le rejet du mélange eau - sédiment à forte concentration induit la naissance de deux tourbillons contrarotatifs de part et d'autre de l'axe du rejet. L'eau environnante se mélange au rejet. Lorsque le jet et les deux tourbillons entre en contact avec le fond la phase de chute se termine pour laisser place à une phase de transport sur le fond. Les phénomènes observés expérimentalement sont bien représenté qualitativement par le modèle à deux phases. La conservation de la masse dans le domaine en cours de calcul est très bonne puisque l'erreur relative est de l'ordre de $0,05 \%$.
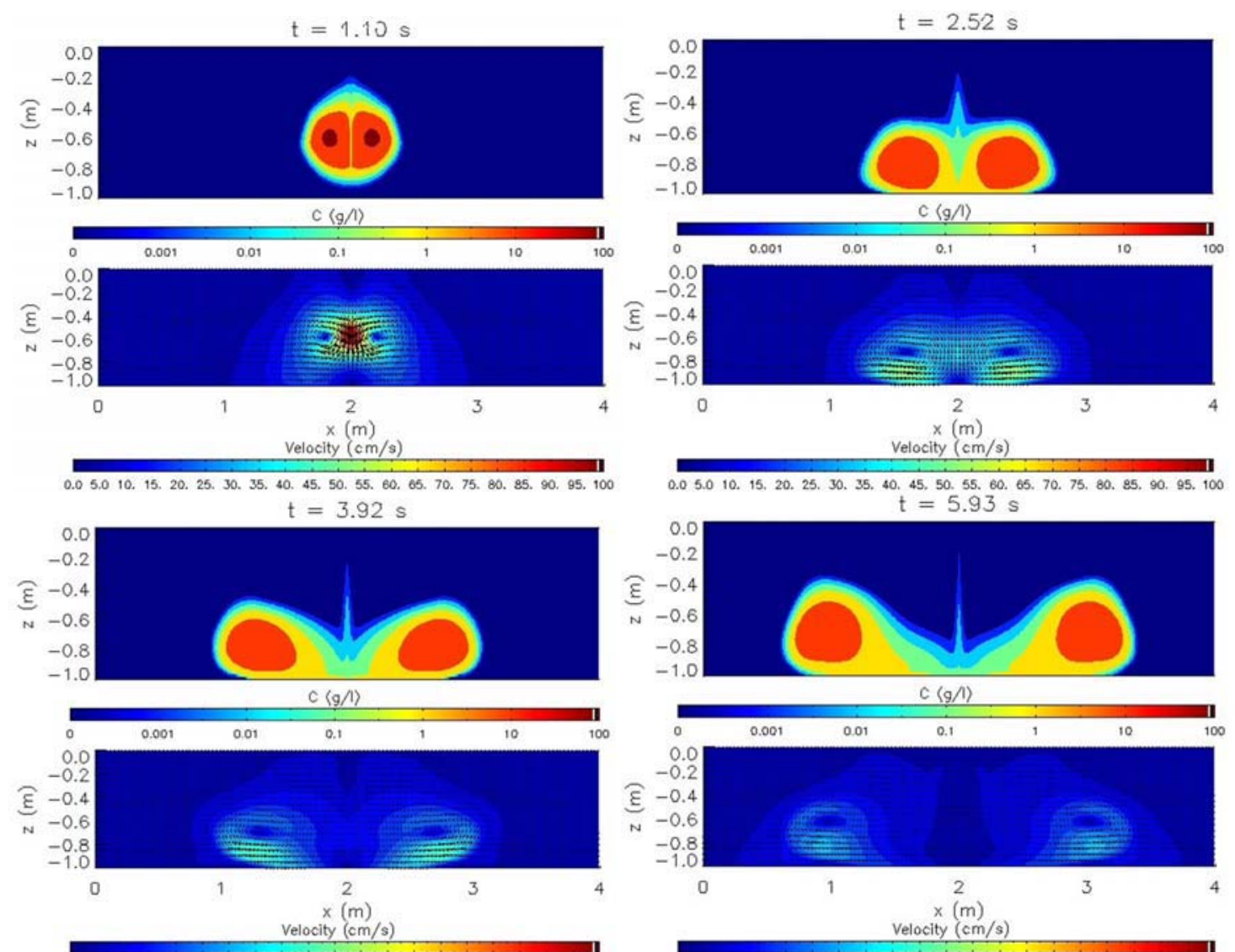

$50,10,15,20,25,30,35,40,45,50,55,60,65,70,75,80,85,90,95,100$

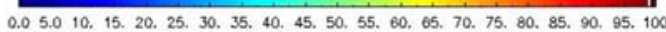

Figure 1. Isocontours de la concentration en sable et champs de vitesse simulés (cas de

l'essai e6) à différents instants. Le temps de chute Tc=1.8 s correspond à l'instant où

l'isocontour à $0,5 \mathrm{~g} / \mathrm{l}$ atteint le fond. On y observe la phase de chute $(t=1.10 \mathrm{~s})$ et la phase de transport sur le fond ( $t=3.92 \mathrm{~s}$ et $t=5.93 \mathrm{~s})$.

Les expériences ont permis de sortir les paramètres suivants : diamètre du rejet $(D r)$, hauteur ou cote du rejet par rapport au fond $(H r)$, temps de chute $(T c)$, vitesse (Ufr) et hauteur $(H f r)$ du front de concentration lors de la phase de progression sur le fond. Comme FRESON (2004) nous avons choisi de considérer le rejet comme les zones de 


\section{XI $I^{\text {èes }}$ Journées Nationales Génie Côtier - Génie Civil}

Les Sables d'Olonne, 22-25 juin 2010

concentrations supérieures ou égales à $C_{c}=0,5 \mathrm{~g} / \mathrm{l}$. Les paramètres précédents sont donc calculés en considérant cette concentration de coupure. Afin de tenir compte de l'incertitude de mesure, les résultats expérimentaux sont tracés en min et max. La figure 2 montre l'évolution de $H r$ et $\mathrm{Dr}$ durant la phase de chute pour les essais e6, e11 et e12. On constate que pour l'essai e6, les résultats sont en bon accord avec l'expérience. Pour les essais e11 et e12, une légère surévaluation de $\mathrm{Hr}$ apparaît tandis que $\mathrm{Dr}$ est légèrement sous-évalué. La diminution du temps de chute et l'augmentation du diamètre $\mathrm{du}$ rejet avec l'augmentation du volume et de la concentration du rejet sont bien reproduits (e6/e11). La diminution du temps de chute et une très faible augmentation du diamètre du rejet avec l'augmentation du diamètre des particules sont également reproduits (e11/e12). Le tableau 2 montre que les résultats de simulation sont proches des données expérimentales. L'erreur relative sur le diamètre de rejet est de l'ordre de $17 \%$. Le temps de chute semble surestimé pour les essais e11 et e12. Concernant la phase de transport sur le fond, la vitesse de propagation est assez bien estimée. Comme sur l'expérience, la vitesse du front augmente avec le volume et la concentration initiale (e6/e11). En revanche, l'influence du diamètre des particules n'est pas bien reproduit (e11/e12). Les écarts sont plus importants sur l'épaisseur du front (Hfr). Ceci montre que la dynamique engendrée dans la phase de transport est trop importante. Le dépôt dans la zone d'impact reste faible. La modélisation des contraintes de cisaillement pourrait être une cause probable de ce défaut.
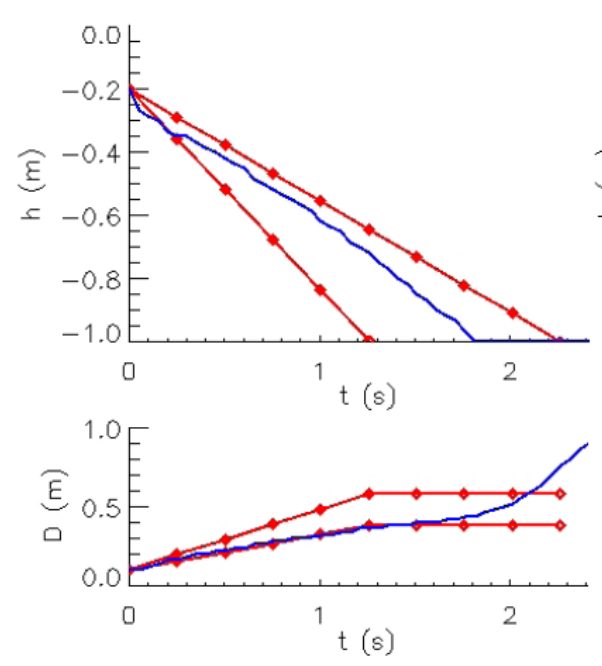
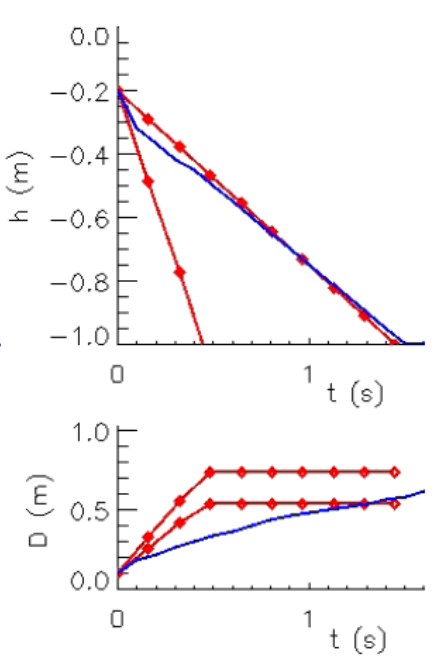
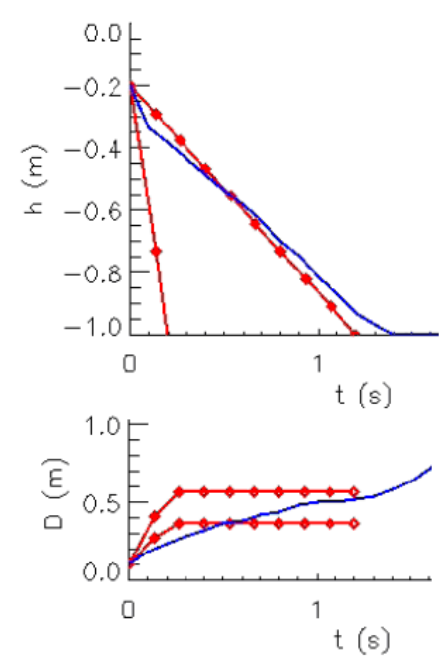

Figure 2. Evolution temporelle de la position inférieure $(\mathrm{Hr})$ et du diamètre $(\mathrm{Dr})$ de rejet durant la phase de chute pour l'essai e6 (gauche), l'essai ell (centre) et l'essai e12 (droite) : - simulation numérique $\leftrightarrow \longleftrightarrow$ expérience de VILLARET et al (1997) en intégrant la barre d'erreur. 
Thème 2 -Dynamique sédimentaire et transports des particules

Tableau 2. Temps de chute (Tc) et diamètre de rejet (Dr) en fin de chute, et Vitesse (Ufr) et hauteur (Hfr) du front du courant de densité durant la phase de transport sur le fond: résultats des simulations (num) et valeurs expérimentales (VILLARET et al., 1997).

\begin{tabular}{|c|c|c|c|c|c|c|c|c|}
\hline essai & $\begin{array}{l}T c_{n u m} \\
(s)\end{array}$ & $\begin{array}{l}T c_{\text {exp }} \\
(s)\end{array}$ & $\begin{array}{l}D r_{\text {num }} \\
\text { (m) }\end{array}$ & $\begin{array}{l}D r_{e x p} \\
(m)\end{array}$ & $\begin{array}{l}U f r_{\text {num }} \\
(\boldsymbol{m} / \mathrm{s})\end{array}$ & $\begin{array}{l}U f r_{\text {exp }} \\
(\boldsymbol{m} / \mathrm{s})\end{array}$ & $\begin{array}{l}H f r_{n u m} \\
(m)\end{array}$ & $\begin{array}{l}H f r_{\text {exp }} \\
\text { (m) }\end{array}$ \\
\hline$e 6$ & 1.8 & $1.76 \pm 0.5$ & 0.54 & $0.48 \pm 0.1$ & 0,18 & 0,17 & 0,43 & 0.2 \\
\hline ell & 1.5 & $0.95 \pm 0.5$ & 0.56 & $0.64 \pm 0.1$ & 0,23 & 0,19 & 0.45 & 0.38 \\
\hline el2 & 1.4 & $0.77 \pm 0.5$ & 0.54 & $0.46 \pm 0.1$ & 0,23 & 0,23 & 0.43 & 0.24 \\
\hline
\end{tabular}

\section{Conclusions}

Cette étude a montré que l'application d'un modèle diphasique à deux fluides au cas du clapage en eau calme de matériaux non-cohésif (sable) reproduisait de manière satisfaisante les résultats expérimentaux. Certains biais (structure verticale du courant de turbidité) restent encore à améliorer avant de passer à l'étape suivante du clapage en présence d'un courant ambiant (La figure 3 présente des résultats de calculs préliminaires dans ce cas) et surtout le clapage de matériaux sablo-vaseux. Une étude particulière du courant de densité devrait permettre de lever ces biais.

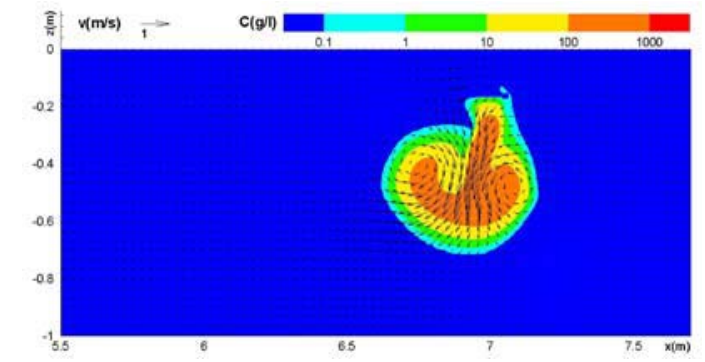

a)

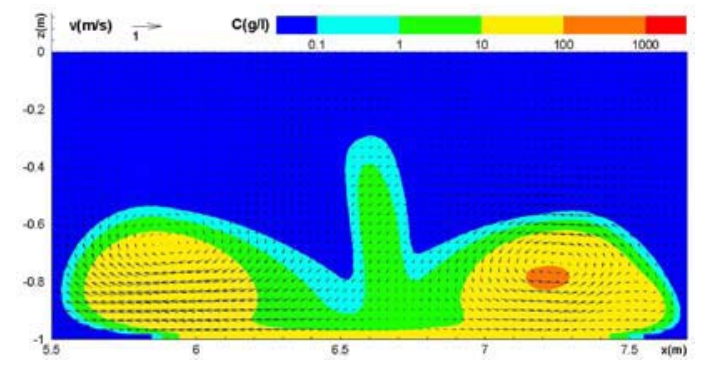

c)

Figure 3. Isocontours de la concentration en sable et champs de vitesse simulés dans la configuration de l'essai 11 avec un courant de $10 \mathrm{~cm} / \mathrm{s}$ (essai e13) à $1 \mathrm{~s}$ (a), 2,1 s (b) et $4 \mathrm{~s}$ (c). L'impact du courant sur la phase de chute est notable. 


\section{Remerciements}

Les auteurs remercient le CETMEF pour le financement de cette étude (contrat $\mathrm{N}^{\circ}$ 05-510006-000-228-6034) et le CRIHAN pour les moyens de calculs.

\section{Références bibliographiques}

BARBRY N., GUILLOU S., NGUYEN K.D. (2000). Une approche diphasique pour le calcul du transport sédimentaire en milieux estuariens. C.R. Acad. Sci., IIb, 328, pp 793-799.

BOUTIN R. (2000). Dragage et rejets en mer. Les produits de type vase. Presses de l'ENPC. 2000, 307 p.

CHAUCHAT J., GUILLOU S. (2008). On turbulence closures for two-phase sedimentladen flow models. J. Geophys. Res., 113, C11017. doi:10.1029/2007JC004708

CHAUCHAT J., GUILLOU S., NGUYEN K.D. (2008). Utilisation des mesures rhéométriques pour la modélisation diphasique du transport sédimentaire. Rapport de contract $\mathrm{N}^{\circ} \mathrm{H} 054$, Université de Caen-CETMEF, 62 p.

FAROUT-FRESON I., SERGENT P., LEFRANÇOIS E., DHATT G. (2006), Modèle numérique de clapage - phase de chute, IX ${ }^{\mathrm{èmes}}$ JNGCGC, Brest, pp 179-186. doi:10.5150/jngcgc.2006.018-F

FRESON F.I. (2004). Simulation numérique du clapage en mer: Etude du champ proche-Chute et Transport sur le fond. Thèse, Université Techn. de Compiègne, $316 \mathrm{p}$.

GARAPON A., VILLARET C., BOUTIN R. (2002). 3D numerical modelling of sediment disposal, Proceedings of the Physics of Estuarine. PECS, $13 \mathrm{p}$.

GRAHAM A.L. (1981). On the viscosity of suspensions of solid spheres. Appl. Sci. Res., 37, pp 275-286. doi:10.1007/BF00951252

GUILLOU S., NGUYEN K.D. (1999). An improved technique for solving twodimensional shallow water problems. Int. J. Numer. Methods Fluids, 29, pp 465-483. doi:10.1002/(SICI)1097-0363(19990228)29:4<465::AID-FLD797>3.0.CO;2-H

GUILLOU S., BARBRY N., NGUYEN K.D. (2000). Calcul numérique des ondes de surface par une méthode de projection et un maillage eulérien adaptatif. C.R. Acad. Sci., IIb, 328, pp 875-881.

HAIDER A., LEVENSPIEL O. (1989). Drag coefficient and terminal velocity of spherical and non-spherical particles. Powder Technol., 58, pp 63-70. doi:10.1016/0032-5910(89)80008-7

HSU T., JENKINS J.T., LIU P. L.-F. (2003). On two-phase sediment transport: Dilute flow. J. Geophys. Res., 108(C3), 3057. doi:10.1029/2001JC001276

KRISHNAPPAN B.G. (1975), Dispersion of granular material dumped in deep water, in Scientific series, Environment Canada, 55, 113 p.

LUNDGREN T. (1972). Slow flow through stationary random beds and suspensions of spheres. J. Fluid Mech., 51, pp 273-299. doi:10.1017/S002211207200120X 
Thème 2 -Dynamique sédimentaire et transports des particules

NGUYEN K.D., GUILLOU S., CHAUCHAT J., BARBRY N. (2009). A two-phase numerical model for suspended-sediment transport in estuaries. Advances in Water Resources, 32, pp 1187-1196. doi:10.1016/j.advwatres.2009.04.001

TEISSON C., SIMONIN O., GALLAND J.C., LAURENCE D. (1992). Turbulence and mud sedimentation: A Reynolds stress model and a two-phase flow model. in Proceedings of 23rd ICCE, ASCE, pp 2853-2866.

VILLARET C., LEKIEN M., CLAUDE B., VINET V. (1997). Etude expérimentale de la dispersion des rejets par clapage d'un mélange de sable et de vase. He-42/97/072/a, LNHE, EDF.

VILlARET C., ClAUDE B., DU RIVAU J.D. (1998). Etude expérimentale de la dispersion des rejets par clapage. He-42/98/065/a, LNHE, EDF. 